\title{
In situ Imaging and Characterizing the Matrix of Extracellular Polymeric Substances (EPS) of Biofilms.
}

\author{
Alan W. Decho
}

Microbial Interactions Laboratory, Department of Environmental Health Sciences, Arnold School of Public Health, University of South Carolina, Columbia, SC. 29208. USA.

The microbial cells of biofilms are surrounded within a matrix of secreted extracellular polymeric substances (EPS)[1]. Polysaccharides, proteins, glycoproteins, proteoglycans, lipids, and even DNA are major molecules that have been isolated from EPS, and contribute to its properties. The matrix, however, is complex and constantly changing. The composition, density and arrangements of adjacent EPS molecules, are known to vary over microspatial (micrometer to nanometer) scales. This se are ultimately important to the properties and functions that EPS afford to the greater biofilm. Physical/ chemical characterization of bulk EPS removes crucial microspatial information. However, microspatial characterization of EPS in situ, or even within laboratory cultures, has been problematic.

Our laboratory has been investigating the cells and EPS matrix of photosynthetic microbial mats using confocal (CSLM) and multiphoton (MP-SLM) scanning laser microscopy, Raman microspectroscopy, and AFM to decipher aspects of the in situ properties of the EPS matrix. Fluorescent lectin probes (for EPS) and oligoprobes (for cells) have enhanced imaging of mat biofilms (Fig. 1), and are indicating that clusters of microbial cells may 'engineer', and perhaps maintain to lesser or greater extents, the extracellular architecture of biofilms. Lectin probing has shown the presence of spatially heterogeneous EPS 'microdomains' [1]. Concurrent FT-IR analyses of bulk EPS extracts have define primary functional groups involved in binding, gelation and precipitation within the EPS matrix (Fig. 2). AFM imaging of selected EPS gels [2] has allowed a first glimpse into the ultrastructural interactions of EPS (Fig. 3) molecules (under controlled conditions), and how bound and unbound water is localized within the highlyhydrated matrix [2]. Finally, the potential for 'glass formation' [3] within EPS during desiccation is being investigated. The role(s) of EPS glass in preserving the activities of important extracellular molecules that are utilized by bacteria upon rehydration has been demonstrated in the laboratory. The involvement of steric changes in amphiphilic polymers is under investigation in this regard. Future studies are planned using cryo-TEM to examine the high-resolution structure of polymeric fibrils of EPS.

\section{References}

[1] Lawrence J.R., et al. 2007. Can. J. Microbiol. 53: 450-458.

[2] Decho, A.W. 1999. Carbohydr. Res. 315: 330-333.

[3] Crowe, J.H. et al. 1998. Annu. Rev. Physiol. 60: 73-103.

[4] This work is supported by grants from the National Science Foundation (CHE-0526821; EF0723707) 
Fig. 1. (A) CSLM image showing X-section of marine microbial mat. Blue (circular) objects are calcium carbonate sand grains, called ooids; green layer are a dense layer of sulfate-reducing bacteria (oligoprobed, individual cells not visible) at mat surface; red stringy cells are cyanobacteria (autofluorescence). (B) Higher magnification image showing carbonate ooids (blue), cyanobacteria (red) and dense EPS gel matrix (green) stained with lectin probe.

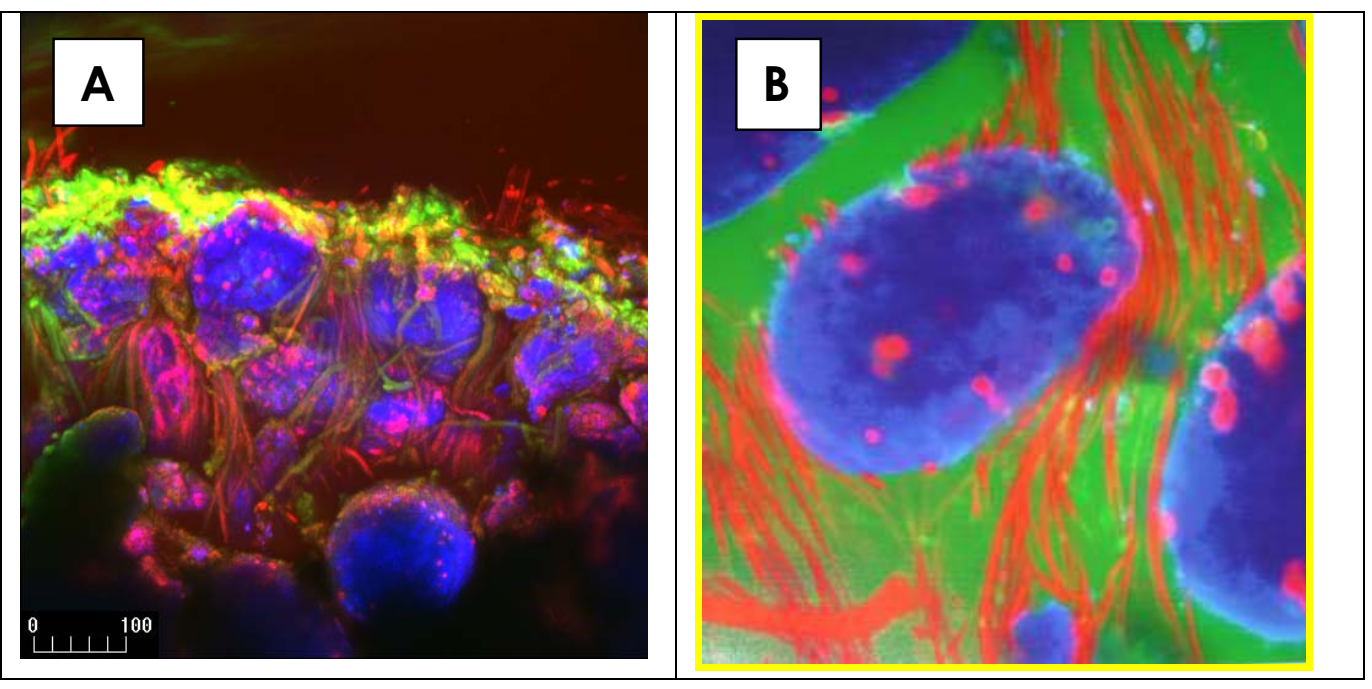

Fig. 2. (A) FT-IR spectra of natural EPS extracted from microbial mat surface. Peaks correspond to specific chemical bonds, and/or functional groups on EPS. (B) Atomic-force micrograph of EPS gel in seawater showing tangled-network structure of molecules in gel, and hydration spaces (for water).

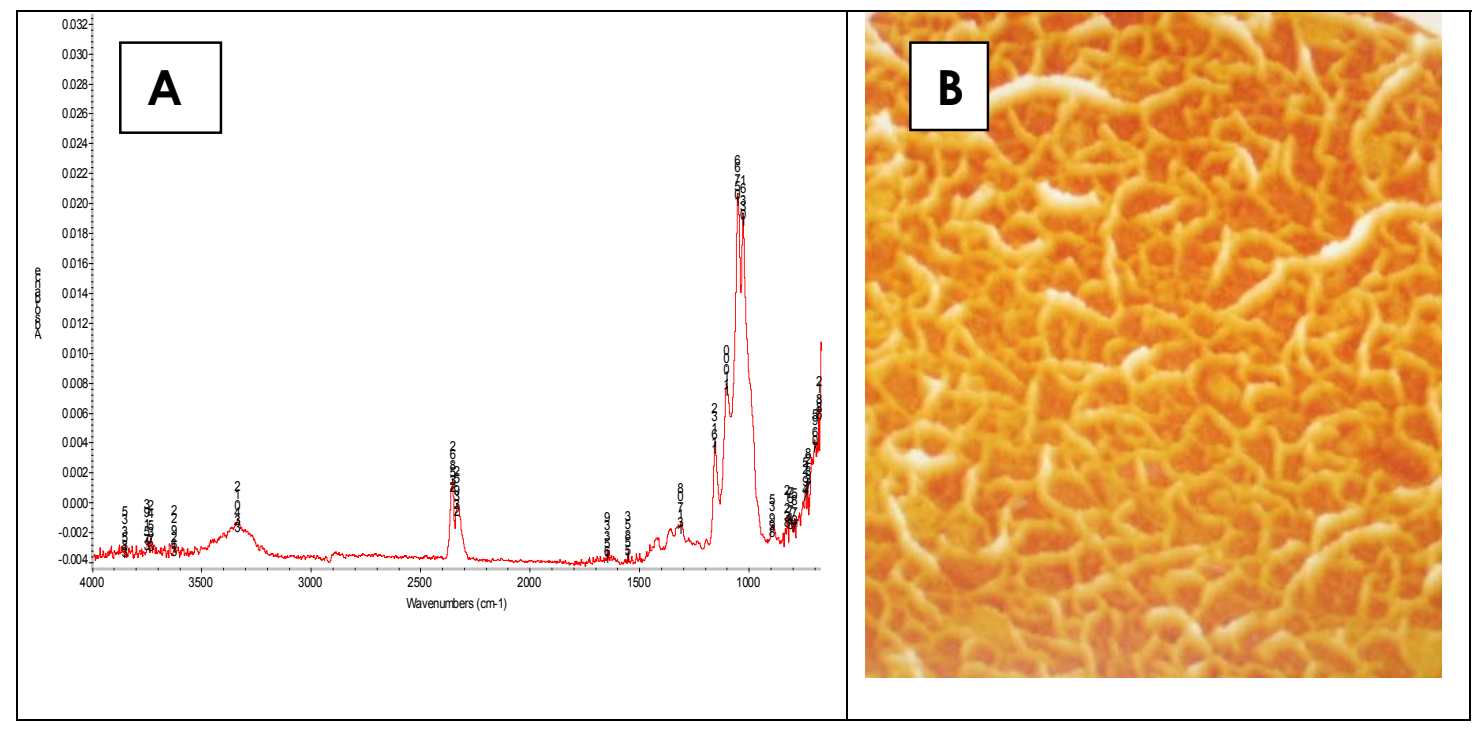

